\title{
2010-A-73-EHSG
}

\section{Antimicrobial susceptibility pattern of Helicobacter suis strains}

\author{
Miet Vermoote*, Frank Pasmans, Bram Flahou, Kim Van Deun, Richard Ducatelle, Freddy \\ Haesebrouck
}

Department of Pathology, Bacteriology and Avian Diseases, Faculty of Veterinary Medicine, Ghent University, Merelbeke, Belgium

Helicobacter suis is a very fastidious porcine gastric pathogen, which is also considered to be of zoonotic importance. In vitro antimicrobial susceptibility can not be determined using standard assays, as this agent only grows in a biphasic medium with an acidic $\mathrm{pH}$. Therefore, a combined agar and broth dilution method was used to analyse the activity of nine antimicrobial agents against nine $H$. suis isolates. After $48 \mathrm{~h}$ microaerobic incubation, minimal inhibitory concentrations (MICs) were determined by software-assisted calculation of bacterial growth. Only for enrofloxacin a bimodal distribution of MICs was demonstrated, indicating acquired resistance in one strain, which showed an AGT $\rightarrow$ AGG $(\mathrm{Ser} \rightarrow \mathrm{Arg})$ substitution at codon 99 of gyrA. In conclusion, the assay developed here is suitable for determination of the antimicrobial susceptibility of $H$. suis isolates, although activity of acid sensitive antimicrobial agents may be higher than predicted from MIC endpoints. 